\title{
Variation of chemosensory receptor content of Campylobacter jejuni strains and modulation of receptor gene expression under different in vivo and in vitro growth conditions
}

Christopher J Day ${ }^{1}$, Lauren E Hartley-Tassell ${ }^{1}$, Lucy K Shewell1, Rebecca M King ${ }^{1}$, Greg Tram¹, Serena K Day², Evgeny A Semchenko ${ }^{1}$ and Victoria Korolik ${ }^{1 *}$

\begin{abstract}
Background: Chemotaxis is crucial for the colonisation/infection of hosts with Campylobacter jejuni. Central to chemotaxis are the group A chemotaxis genes that are responsible for sensing the external environment. The distribution of group A chemoreceptor genes, as found in the C. jejuni sequenced strains, t/p 1-4, 7, 10 and 11 were determined in 33 clinical human and avian isolates.

Results: Group A t/p gene content varied among the strains with genes encoding t/p 1 (aspartate receptor, ccaA) and $t / p 7$ present in all strains tested, where as $t / p 11$ was present in only one of our international collection clinical isolates, C. jejuni 520, but was more prevalent (9/13) in the freshly isolated clinical stains from patients who required hospitalisation due to $\mathrm{C}$. jejuni infection (GCH1-17). Relative expression levels of the group A t/p genes were also determined in C. jejuni reference strains NCTC 11168-GS, $11168-\mathrm{O}$ and 81116 using cells grown in vitro at $37^{\circ} \mathrm{C}, 42^{\circ} \mathrm{C}$ and maintained at room temperature and with cells isolated directly from murine and avian hosts by immune magnetic separation without subsequent culture. Gene expression of t/p genes was varied based on strain, growth conditions and in vivo isolation source. T/p1, although the most conserved, showed the lowest and most varied mRNA expression and protein production under laboratory conditions. T/p7 was highly expressed at most conditions tested, and gene expression was not influenced by the t/p7 gene encoding a full length protein or one expressed as separate periplasmic and cytoplasmic domains.
\end{abstract}

Conclusion: We have shown that chemosensory receptor set variation exists among C. jejuni strains, but is not dependent on the isolation source.

Keywords: Chemotaxis receptor, Campylobacter, Transducer-like proteins

\section{Background}

Campylobacter jejuni is a causative agent of acute bacterial gastroenteritis in humans, and is responsible for an estimated 500 million cases annually worldwide $[1,2]$. Although this bacterium poses a significant economic burden, little is known or understood about the mechanisms of pathogenicity. Some factors, however, have been ascertained to contribute toward the overall

\footnotetext{
* Correspondence: v.korolik@griffith.edu.au

'Institute for Glycomics, Griffith University Gold Coast Campus, Griffith

University, Griffith, Australia

Full list of author information is available at the end of the article
}

pathogenicity of the infecting strain such as chemotaxis, adherence to host cells and surface glycans including lipooligosaccharide [3].

Chemotaxis and motility have been implicated in the colonisation and virulence of many pathogenic bacteria such as Escherichia coli, Salmonella enterica serovar Typhimurium, as well as C. jejuni [3,4]. Homologues of the chemotactic pathway have been identified in C. jejuni NCTC 11168 and include ten putative chemotactic sensory receptors, Tlps, and two aerotaxis receptors [5]. The receptors are grouped according to their putative function as assigned by homology to known chemoreceptors

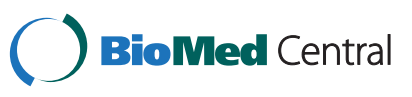


of other organisms [5,6]. The group A consist of Tlp1, 2, $3,4,7$ and 10, all of which contain distinct domains comprising of two transmembrane domains, a sensory domain and a highly conserved cytoplasmic domain [5]. Due to similarity to methyl-accepting chemotactic proteins from other bacterial species, group A Tlp receptors are thought likely to sense ligands external to the cell [5]. Only two of the group A Tlp proteins of $C$. jejuni have been characterised to date, the aspartate receptor, Tlp1 [7] and Tlp7 which binds to formic acid [8].

Recent analysis of full and partial sequenced strains of C. jejuni has shown diversity in the group A Tlp receptor set and indicated that Tlp1 was the only receptor universally represented in all sequenced strains of C. jejuni [6]. This high conservation can be explained by the fact that tlp1 encodes the aspartate receptor for C. jejuni [7], aspartate being one of the carbon sources used in C. jejuni metabolism. The receptor set for 81116 was previously reported to be similar to that of 11168 genome sequenced strain, including that of Tlp7, which is represented as a "pseudogene", however, Tlp7 is presumed to be a functional protein in strain HB93-13, as there is no stop codon to interrupt the sequence [6]. A recent study has shown that each portion of $t l p 7$ can be translated as separate proteins and still function in chemotaxis of this organism [8].

It has previously been suggested that receptor subset variation may be dependent on strain source or relative pathogenicity, since variance in the chemoreceptor subset has been shown for some uropathogenic strains of E. coli, which all lack the functional receptors Trg (ribose and galactose) and Tap (dipeptides) usually present within strains isolated from faecal material [9]. In C. jejuni, tlp7 is the only receptor where this has been tested using strains from different sources. Zautner et al. (2011) showed that $d t l p 7, t l p 7$ encoded by two separate genes rather than a single transcript, was overrepresented in bovine strains and underrepresented in human isolates [10].

In addition to 6 group A tlp genes encoded by C. jejuni 11168, a unique tlp, designated as Tlp11, was identified in some $C$. jejuni strains and was shown to share sequence similarity with TcpI, a chemoreceptor involved in stimulating the expression of the $\mathrm{CT}$ and TCP pathway of Vibrio cholerae [6]. It has yet to be established if Tlp11 exists in other $C$. jejuni isolates and whether it has a role in enhancing virulence or if it has an effect on the expression levels of the other group A tlp genes.

Although genome analysis has demonstrated which receptor sets are present in partially and fully-sequenced strains of $C$. jejuni, whether gene expression is conserved has yet to be elucidated. Here we report the variation in C. jejuni chemoreceptor gene subsets within the genomes of $33 \mathrm{C}$. jejuni strains, including NCTC 11168 -GS and -O, isolated from both avian and human hosts. C. jejuni 11168-GS is the non-colonising, non-invasive variant of NCTC 11168 with known decreases in virulence-associated phenotypes and with a number of point mutations when compared to the original isolate (11168-O) from which it was derived [11]. We also report receptor gene expression modulation in vivo, during colonisation of avian and mammalian hosts, and in vitro under varying growth conditions.

\section{Results}

\section{Tlp gene content of different $C$. jejuni strains}

Thirty-three strains of $C$. jejuni isolated from chicken and human hosts were analysed to elucidate which $t p$ genes were present in the genomes of these strains and if any relatedness to the isolation host could be ascertained. The identity of each group A Tlp receptor for all seven known group A tlp genes, tlp1-4, 7, 10 and 11 in each of the $33 \mathrm{C}$. jejuni strains were determined by PCR amplification (Table 1). The C. jejuni strains tested appeared to possess varied sets of group A Tlp receptor genes, with six strains $(C$. jejuni 520, GCH3, 6, 10, 14 and 17) possessing all seven group A tlp genes (Table 1). Tlp1 was present in all strains tested and is the only universally conserved $t l p$ gene within the strains (Table 1 ). Tlp7 was present in 31 of 33 strains, while, tlp10 and tlp3 were detected in 30 of 33 strains making them the next most conserved of the tlp genes (Table 1). The least representatively conserved tlp genes, other than tlp11, were $t l p 2$ and tlp4 (Table 1).

\section{Sequencing results of $t \mid p 7$}

Tlp7 is annoted as a "pseudogene" in C. jejuni 11168 though a recent study showed it is functional in strains that do not possess an uninterrupted $t l p 7$ reading frame [8]. Another study also showed that the presence of the interrupted reading frame is over or underrepresented in strains isolated from different sources [10]. Due to this we sequenced each $t l p 7$ amplicon to determine if the gene was present as a full length reading frame or if it was split into two open reading frames with the introduction of a stop codon. The PCR primers used to amplify $t l p 7$ were designed to amplify across the split between Cj0951c/Cj0952c of C. jejuni 11168. Sequencing data showed in 23 of the 31 strains that contain $t l p 7$ that it is present as an uninterrupted gene sequence (Table 1). In the remaining eight strains it exists as two separate open reading frames indicating that Tlp7 is produced as two separate proteins in these strains (Table 1).

\section{Relative expression of t/p genes by qPCR}

In order to determine relative gene expression profiles of the $C$. jejuni group A $t l p$ genes at varying conditions 
Table 1 Results of PCR amplification of t/p genes of C. jejuni strains isolated from both chickens and humans \begin{tabular}{llllllll}
\hline C. jejuni strain & Tlp1 & Tlp2 & Tlp3 & Tlp4 & Tlp7 & Tlp10 & Tlp11
\end{tabular}

\begin{tabular}{llllllll}
\hline \multicolumn{2}{l}{ Chicken isolates } & & & & & & \\
008 & + & - & + & + & $+^{\mathrm{P}}$ & + & - \\
019 & + & - & + & - & $+^{\mathrm{P}}$ & + & - \\
108 & + & - & + & + & $+^{\mathrm{P}}$ & + & - \\
331 & + & + & - & + & $+^{\mathrm{w}}$ & + & - \\
434 & + & - & + & + & $+^{\mathrm{w}}$ & + & - \\
506 & + & - & + & + & $+^{\mathrm{w}}$ & - & - \\
913 & + & + & + & - & $+^{\mathrm{w}}$ & - & -
\end{tabular}

Human isolates Laboratory maintained

\begin{tabular}{|c|c|c|c|c|c|c|c|}
\hline 173 & + & - & + & + & $+w$ & + & - \\
\hline 11168-GS & + & + & + & + & $t^{P}$ & + & - \\
\hline $11168-0$ & + & + & + & + & $+^{\mathrm{P}}$ & + & - \\
\hline 351 & + & + & + & - & $+w$ & + & - \\
\hline 430 & + & + & + & + & $+w$ & + & - \\
\hline 435 & + & + & + & + & $+{ }^{w}$ & + & - \\
\hline 440 & + & + & + & + & $+w$ & + & - \\
\hline 520 & + & + & + & + & $+{ }^{w}$ & + & + \\
\hline 705 & + & + & + & - & $+{ }^{w}$ & + & - \\
\hline 8 & + & - & + & + & $+{ }^{w}$ & + & - \\
\hline 81116 & + & + & + & + & $+w$ & + & - \\
\hline 81-176 & + & + & - & + & $+w$ & + & - \\
\hline 93 & + & + & + & + & $+w$ & - & - \\
\hline \multicolumn{8}{|c|}{ Human isolates Fresh clinical isolates } \\
\hline GCH1 & + & + & + & + & $+^{P}$ & + & - \\
\hline GCH2 & + & + & + & + & $+^{P}$ & + & - \\
\hline GCH3 & + & + & + & + & $+{ }^{w}$ & + & + \\
\hline GCH4 & + & - & + & + & $+w$ & + & + \\
\hline GCH5 & + & + & + & + & $+{ }^{w}$ & + & - \\
\hline GCH6 & + & + & + & + & $+{ }^{w}$ & + & + \\
\hline GCH7 & + & + & + & - & - & + & + \\
\hline GCH9 & + & + & + & + & $t^{P}$ & + & - \\
\hline GCH10 & + & + & + & + & $+{ }^{w}$ & + & + \\
\hline GCH11 & + & - & - & - & $+w$ & + & + \\
\hline GCH14 & + & + & + & + & $+{ }^{w}$ & + & + \\
\hline GCH15 & + & + & + & - & - & + & + \\
\hline GCH17 & + & + & + & + & $+w$ & + & + \\
\hline
\end{tabular}

$+=$ Positive PCR product present in repeat experiments.

- = No product detected in repeat PCR amplifications.

$+{ }^{\mathrm{P}}$ refers to the presence of tlp7 as two separately co-expressed genes.

$+{ }^{\mathrm{W}}$ refers to a whole gene able to be translated into a complete protein product. Sequencing was performed in triplicate to ensure accuracy of the results.

in vitro and in vivo, C. jejuni strains, 11168-GS, 11168-O and 81116 were grown in vitro, at $37^{\circ} \mathrm{C}, 42^{\circ} \mathrm{C}$ and maintained in pond water at $20-25^{\circ} \mathrm{C}$, and in vivo by colonising avian and mammalian hosts and then isolated directly from animals by immunomagnetic separation (IMS) (Methods). Growth at $37^{\circ} \mathrm{C}, 42^{\circ} \mathrm{C}$ was assessed as it mimics mammalian and avian hosts in vitro and allows a direct comparison with expression of Tlps in cells directly isolated from animal hosts. Maintenance in pond water (from local farm pond, sterilised) at $20-25^{\circ} \mathrm{C}$ is used to mimic environmental conditions [12], as surface and reservoir water contamination is a potential environmental source for C. jejuni outbreaks [13-16]. Relative gene expression of the group A tlp receptors in C. jejuni under all these different conditions was then assessed by Quantitative PCR. The expression of $t l p$ genes was compared between each strain and growth condition. Only statistically significant differences $(\mathrm{p}<0.05)$ are described below.

\section{Comparison of the group A tlp gene expression for C. jejuni 11168-O, 11168-GS and 81116}

The expression levels of $t l p$ genes within $C$. jejuni strain $11168-\mathrm{O}$ were generally varied, with $t l p 7$ and 10 showing higher expression levels compared to the other tlp genes. It is interesting to note that tlp1 showed the lowest level of expression (Figure 1), particularly in cells isolated from the intestines of chicks and from bacteria grown in laboratory conditions at $42^{\circ} \mathrm{C}$. Contrary to all expectations, the expression of $t l p 7$ was very high under all conditions tested, irrespective of the fact that it is a present as two separate gene transcripts in C. jejuni 11168-O (Figure 1). This high level of expression correlated with the finding that $t l p 7$ may act as a functional receptor even when present as two separate genes [8].

In contrast, the expression profiles for the group A $t l p$ genes in C. jejuni 11168-GS all displayed similar patterns of gene expression. The expression of tlp genes in 11168-GS appeared to be temperature dependent with the lowest level of expression observed in bacteria maintained at room temperature in pond water (Figure 2). The highest levels of expression were observed in bacteria grown at $37^{\circ} \mathrm{C}$, while in most cases expression at $42^{\circ} \mathrm{C}$ were lower than those seen at $37^{\circ} \mathrm{C}$. Unlike $C$. jejuni 11168-O, 11168-GS tlp gene expression appears to be related to temperature, however not all tlp genes were expressed at the same level.

Gene expression profiles for the group A $t l p$ genes in C. jejuni 81116 in vitro and in vivo were also diverse. It is notable that the expression of the aspartate receptor gene, tlp1, was the lowest of all tlp genes, with almost no detectable expression when grown at $37^{\circ} \mathrm{C}, 42^{\circ} \mathrm{C}$ or in pond water. In contrast, tlp1 was highly expressed in C. jejuni 81116 isolated from in vivo hosts $(\mathrm{p}<0.05)$ (Figure 3). Expression levels seen for tlp1, tlp2, tlp3, tlp 7 and tlp10 were all higher in C. jejuni isolated from both in vivo hosts, compared to bacteria grown at an equivalent temperature under laboratory conditions, indicating 


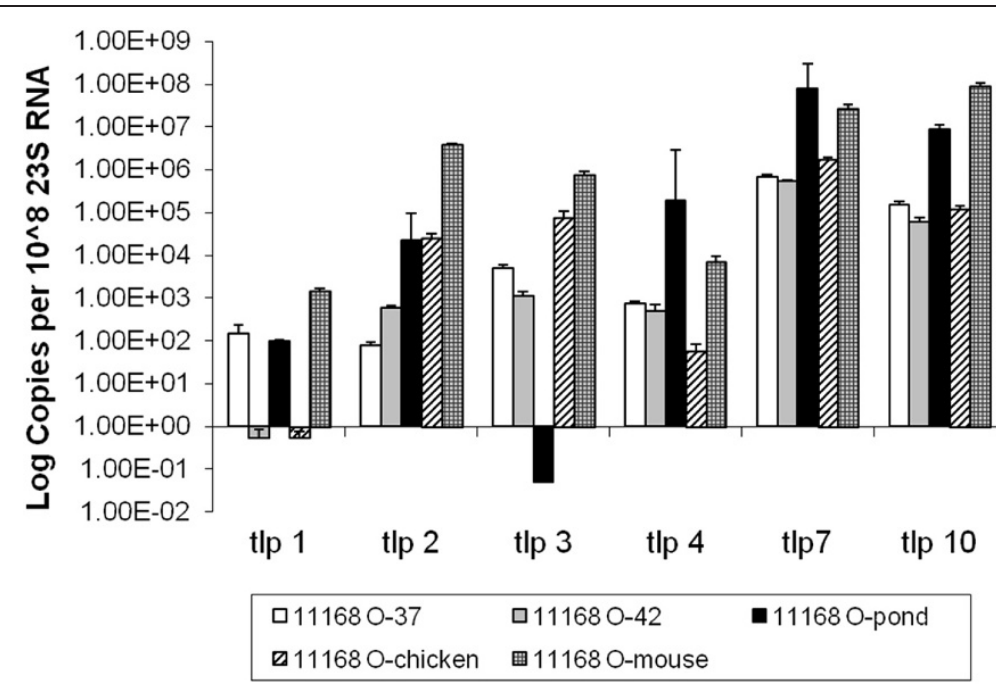

Figure 1 Expression of Group A t/p genes for C. jejuni strain 11168-O. Relative gene expression profiles of Group A t/p genes for C. jejuni $11168-\mathrm{O}$ grown at $37^{\circ} \mathrm{C}, 42^{\circ} \mathrm{C}$, maintained in pond water and isolated in vivo from chicken and mouse. Expression is standardised and the scale is shown in log (copies per $10^{8}$ of $23 \mathrm{~S}$ RNA). 37 : grown under laboratory conditions at $37^{\circ} \mathrm{C}, 42$ : grown under laboratory conditions at $42^{\circ} \mathrm{C}$, pond: maintained in an environmental water source at room temperature, $22^{\circ} \mathrm{C}$, chicken: directly isolated from chicken caecal content by Dyna-beads, mouse: directly isolated from mouse intestines by Dyna-beads. Standard errors are shown as bars above the mean of a minimum of 3 independent PCR reactions.

that host factors are involved in stimulation of $t l p$ gene expression. The expression of $t l p 7$ and 10 were consistently higher than the other tlp genes under all conditions tested, with the highest expression observed for $t l p 7$ in 81116 isolated from the intestines of mice.

\section{Verification of Tlp1 expression by Western blot}

To verify that mRNA levels detected by qPCR reflected the level of protein produced in the bacterial cells,
Western blot analysis was performed, using whole cell protein of C. jejuni 11168-O, 11168-GS and 81116 grown or maintained in the laboratory at room temperature, at $37^{\circ} \mathrm{C}$ and $42^{\circ} \mathrm{C}$, with polyclonal antisera raised against purified periplasmic domain of Tlp1 protein (Figure 4a). Quantitative analysis of the cellular Tlp1 protein, detected by the specific antisera, showed that cellular protein levels changed according to the growth conditions. Tlp1 was present in 11168-O grown

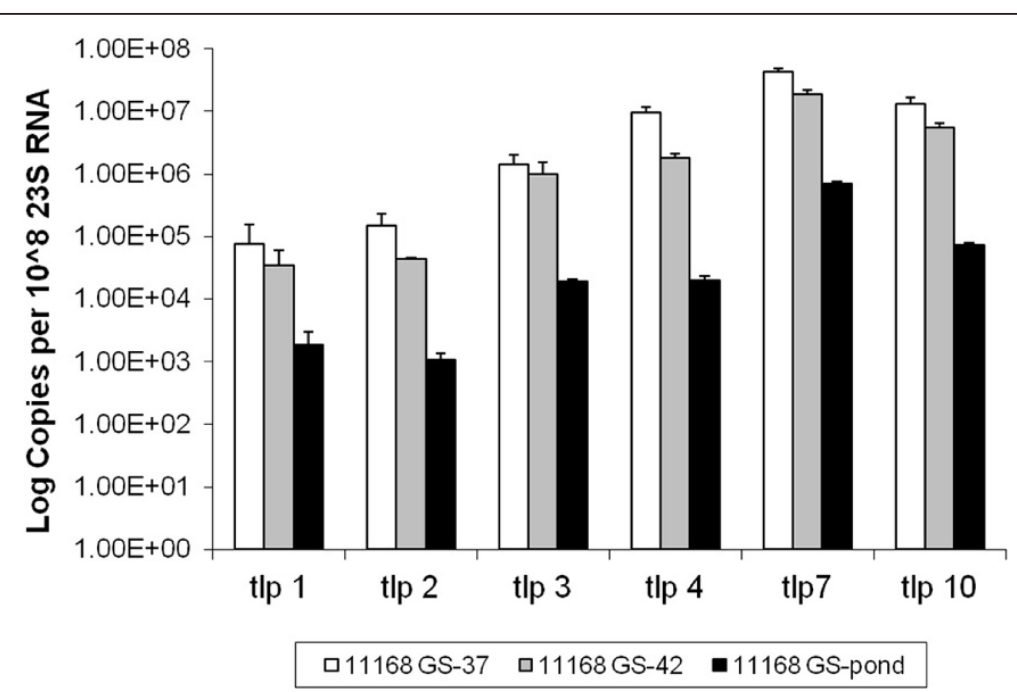

Figure 2 Expression of Group A t/p genes for C. jejuni strain 11168-GS. Relative gene expression profiles of Group A tlp genes for C. jejuni 11168 -GS grown at $37^{\circ} \mathrm{C}, 42^{\circ} \mathrm{C}$ and maintained in pond water. Expression is standardised and the scale is shown in log (copies per $10^{8}$ of $23 \mathrm{~S}$ RNA). 37: grown under laboratory conditions at $37^{\circ} \mathrm{C}, 42$ : grown under laboratory conditions at $42^{\circ} \mathrm{C}$, pond: maintained in an environmental water source at room temperature, $22^{\circ} \mathrm{C}$. Standard errors are shown as bars above the mean of a minimum of 3 independent PCR reactions. 


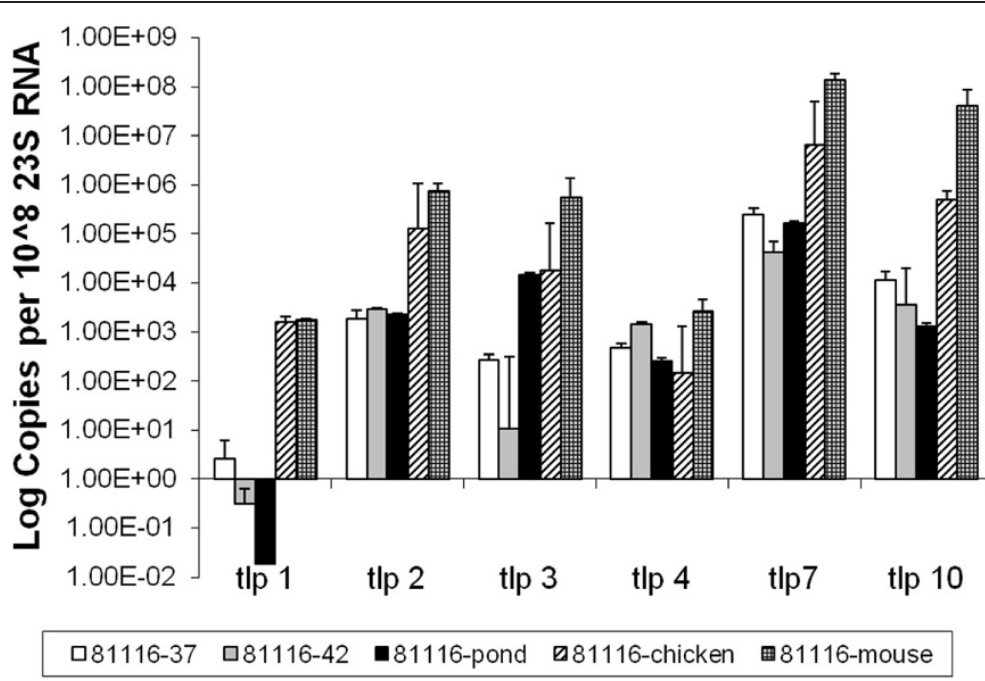

Figure 3 Expression of Group A tlp genes for C. jejuni strain 81116. Relative gene expression profiles of Group A t/p genes for C. jejuni 81116 grown at $37^{\circ} \mathrm{C}, 42^{\circ} \mathrm{C}$, maintained in pond water and isolated in vivo from chicken and mouse. Expression is standardised and the scale is shown in $\log$ (copies per $10^{8}$ of $23 \mathrm{~S}$ RNA). 37 : grown under laboratory conditions at $37^{\circ} \mathrm{C}, 42$ : grown under laboratory conditions at $42^{\circ} \mathrm{C}$, pond: maintained in an environmental water source at room temperature, $22^{\circ} \mathrm{C}$, chicken: directly isolated from chicken caecal content by Dyna-beads, mouse: directly isolated from mouse intestines by Dyna-beads. Standard errors are shown as bars above the mean of a minimum of 3 independent PCR reactions.
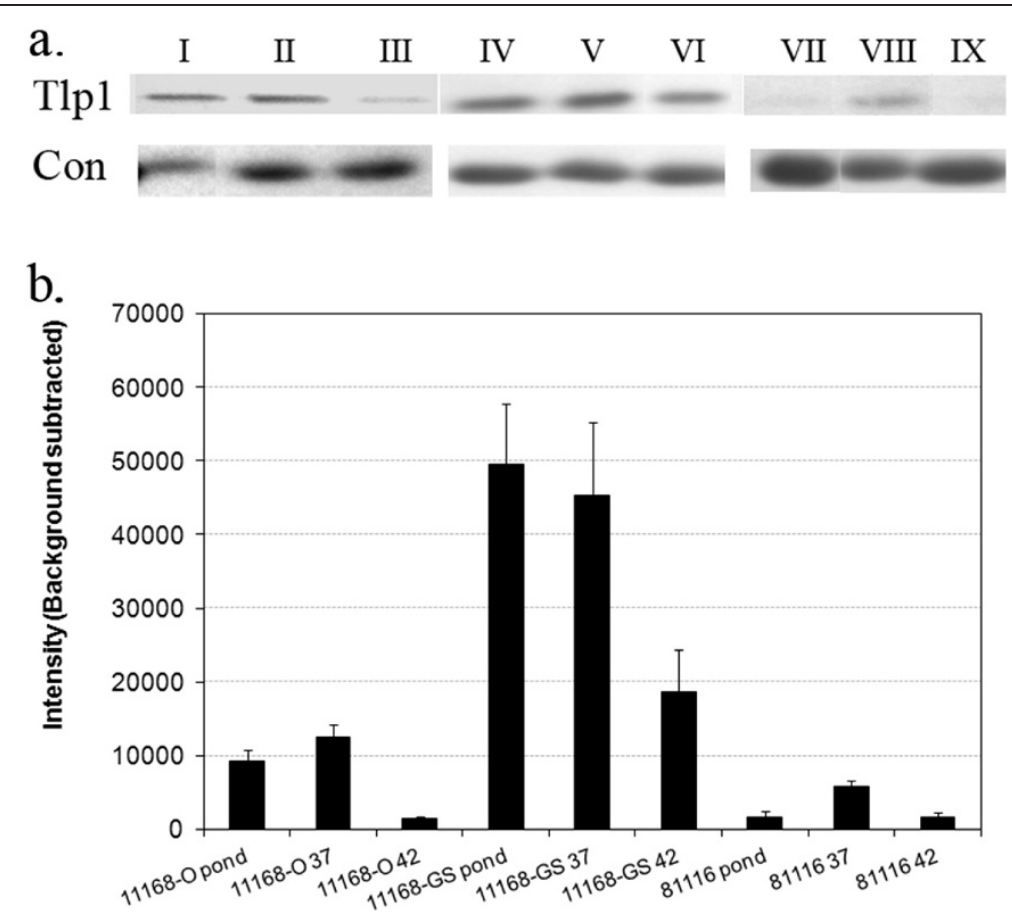

Figure 4 Quantitative protein analysis of cellular Tlp1 levels. a.) Representative blot result from a Western blot performed using anti-Tlp1 sera. Samples are as follows for Tlp1 and Con; I). C. jejuni 11168-O maintained at room temperature in pond water; II). grown at $37^{\circ} \mathrm{C} ; \mathrm{III)}$. grown at $42^{\circ} \mathrm{C}$. IV. C. jejuni $11168-\mathrm{GS}$ maintained at room temperature in pond water; V). grown at $37^{\circ} \mathrm{C}$; VI). grown at $42^{\circ} \mathrm{C}$. VII). C. jejuni $81116 \mathrm{maintained}$ at room temperature in pond water; VIII). grown at $37^{\circ} \mathrm{C}$; IX). grown at $42^{\circ} \mathrm{C}$. A single band was observed at $\sim 75 \mathrm{kDa}$ corresponding to the predicted size of Tlp1. The loading control shows the band $(\sim 30 \mathrm{kDa})$ that was used to ensure the same amount of protein was loaded in each well. b.) Quantitative densitometry analysis of Tlp1 protein detected by anti-TIp1 sera. Average background subtracted band intensity was determined using QuantityOne software (Bio-Rad) from triplicate repeat anti-Tlp1 Western blots of C. jejuni 11168-O, 11168-GS and 81116 maintained at room temperature in pond water; grown at $37^{\circ} \mathrm{C}$; grown at $42^{\circ} \mathrm{C}$. Errors bars equal to $3 \times$ standard error of the mean (SEM). 
at $37^{\circ} \mathrm{C}$ at 1.4 fold greater than in pond water maintained bacteria, and 9.3-fold greater than in bacteria grown at $42^{\circ} \mathrm{C}$ (Figure $4 \mathrm{~b}$ ). These results are in agreement with qPCR analysis which showed that Tlp1 was expressed highest in $C$. jejuni grown at $37^{\circ} \mathrm{C}, 1.5$-fold more than $C$. jejuni maintained in pond water at room temperature and 275-fold higher than $C$. jejuni grown at $42^{\circ} \mathrm{C}$. The protein levels of Tlp1 were seen to be more than four-fold higher in C. jejuni 11168-GS then in any of the conditions tested for C. jejuni 11168-O or 81116 which correlates well with the apparent over-expression seen in 11168-GS for tlp1. C. jejuni 81116 showed the lowest protein levels also in agreement with the expression data.

\section{Discussion}

This report describes the analysis of the group A chemosensory receptor content of various $C$. jejuni strains and the modulation of expression of the tlp genes under varying in vitro and in vivo conditions. Analysis of the chemoreceptor subsets demonstrated that the most conserved $t l p$ genes were $t l p 1$ and $t l p 7$, with the presence of these genes verified in all bacterial strains tested. Previous analysis of the ten sequenced strains (NCBI) revealed that in all strains, tlp1 amino acid sequences were $99-100 \%$ identical [6]. It appears likely that this level of conservation is due to Tlp1 being the sensory receptor for aspartate in C. jejuni [7], where aspartate is one of the few carbon sources utilised in C. jejuni metabolism $[17,18]$. It is interesting to note that although tlp1 was ubiquitously present within $C$. jejuni strains tested, it had the lowest levels of expression in all strains tested at different temperatures in vitro and in bacteria isolated directly from animal hosts. This observation contrasts with reports for other bacterial aspartate receptors, including Tar of E. coli, which is 5-10 fold more abundant than other chemoreceptors in that organism [19]. It would be interesting to determine if Tlp1 is indeed a minor receptor among others or whether there are controlling elements involved in translation and protein stability that may influence the numbers of individual receptors in receptor clusters which are yet to be demonstrated for $C$. jejuni. We can note, however, that expression of the tlp1 gene appears to be tightly controlled for successful colonisation of chickens [7]. In Hartley-Tassell et al. (2009), we showed that an isogenic mutant of tlp1 failed to properly colonise the chick model indicating that expression of tlp1 is involved in establishing normal colonisation. We also showed that over-expression of tlp1 was detrimental to normal colonisation as the complemented isogenic mutant of tlp1 had comparatively higher expression levels than that seen in wild-type C. jejuni $11168-\mathrm{O}$ and thus was only able to poorly complement the mutant [7].
Similar to the aspartate sensory receptor, $t l p 7$ was present in 31 of the 33 strains tested in this study. Tlp7 was previously reported as being a "pseudogene" in C. jejuni 11168 [5] and in all but one of the sequenced strains (NCBI), C. jejuni HB93-13 [6]. However, with the full annotated sequence of C. jejuni 81116 and an updated annotation of $C$. jejuni 81-176 being released, $t l p 7$ has been reassigned as a functional gene in these strains, which agrees with our sequence analysis. Interestingly, $t l p 7$ shows amino acid identity of $>93 \%$ among the strains we tested, irrespective whether the gene was an uninterrupted open reading frame or if it was present as two open reading frames separated by a stop codon. In addition, $t l p 7$ was highly expressed, often being the most abundantly expressed of all group A tlp genes in strains 81116 and NCTC 11168 (both -GS and -O) which were tested using different growth conditions, including expression in vivo in murine and avian hosts. It has been shown that the two proteins of Tlp7, Cj0951c and Cj0952c, are expressed separately but can still function as a formic acid receptor [8]. This indicates that the periplasmic and cytoplasmic domains of Tlp7 encoded by Cj0951c/Cj0952c are likely to be able to integrate into sensory receptor clusters and interact in order to transduce the signal to the $\mathrm{CheAY} / \mathrm{CheW} / \mathrm{CheV}$ complex $[7,8]$.

The second most commonly occurring chemoreceptors were tlp3 and tlp10. Tlp3 was absent in 81-176, 331 and GCH11 but showed highly variable expression depending on the strain of bacteria and the growth/ maintenance condition tested. Expression of tlp10 was high in all strains at most of the conditions tested. Although no ligand has been identified for Tlp10 in C. jejuni, the high overall expression of tlp10 indicates that the ligand may be of some importance for the survival or colonisation of this organism. Expression of this receptor gene within an animal host, in particular the murine model, was higher than under laboratory conditions, with 100-1000 fold greater expression in mice than in chickens indicating a possible role of tlp10 in opportunistic infection of mammalian hosts.

The presence of $t l p 2$ and 4 within the genomes of C. jejuni were the most variable with 13 strains lacking one or both of these genes. This result is comparable to the analysis of the sequenced strains of $C$. jejuni (NCBI) with four of the 10 strains lacking one or both of $t l p 2$ or 4. Like Tlp3, the amino acid sequences of Tlp2 and 4 are less conserved than Tlp1 and 10. The expression levels of tlp 2 and $t l p 4$ were variable between strains and conditions tested with $t l p 2$ being one of the most abundantly expressed tlps in C. jejuni 11168-O isolated from mice. Little is known about either Tlp2 or Tlp4 with respect to ligand binding specificity; however it is interesting to note that these two Tlps along with Tlp3 share almost 
$100 \%$ homology within the cytoplasmic signalling domain of the proteins [5].

Interestingly one of the recently acquired hospital isolates, GCH11, lacked all three of these tlps (tlp2, 3 and 4). This strain only possessed $t l p 1,7^{w}, 10$ and 11 and was able to produce disease of sufficient severity to require hospitalisation. While no data is available on the age or immune competency of the patient, it is clear that a strain with this subset of receptors is able to efficiently infect a human host and cause disease. In 11168-O and 81116, tlp1, 7 and 10 were all induced when in an animal host as compared to laboratory growth conditions. The regulation of tlp11 under host conditions is currently unknown.

Tlp11 was the least common of the group A tlps, only present in the genome of ten of the 33 strains tested and only found in one of the 10 sequenced strains of C. jejuni, 84-25. The expression of tlp11 did not vary with the conditions tested. As yet the ligand for Tlp11 is unknown but interestingly $C$. jejuni $84-25$ is an isolate from a rare Campylobacter meningitis case [20], while 520 is a highly invasive strain of C. jejuni [6] and each of the Gold Coast Hospital isolates were of sufficient disease severity that the infected individuals required hospitalisation. Thus suggesting that Tlp11 may in fact be a marker of virulence in C. jejuni.

It is important to note that C. jejuni 11168-GS and 11168-O express group A $t l p$ genes differently under the same conditions, with 11168-GS generally expressing the tlps at a higher and more uniform level than 11168O. A representative example of this difference was the expression of $t l p 1$ at growth temperatures of $37^{\circ} \mathrm{C}$ and $42^{\circ} \mathrm{C}$ with $\mathrm{C}$. jejuni 11168-GS expressing tlp1 up to 10,000 fold greater than 11168-O. The protein level of Tlp1 in C. jejuni 11168-GS was also shown to be significantly higher than that seen for 11168-O. Gaynor et al. (2004) noted changes in sigma factor sequences indicating that there may be changes in the way genes are regulated in the two strains [11]. While their analysis did not discover any expression changes in tlps there was expression changes in genes involved in chemotaxis, such as CheW and flagella. In addition, there were differences noted in amino acid uptake and catabolism genes including some involved in the processing of aspartate [11]. The comparison of data presented here and that already shown by Gaynor et al. (2004) indicates that there is likely to be a broad disregulation of chemotaxis and the processing of the molecules that are known to be ligands for C. jejuni chemotaxis in 11168-GS. This disregulation may be directly related to the protein sequence changes noted in the three sigma factors screened [11]. As we have previously mentioned, tight control of tlp1 expression appears to be important for optimum colonisation of chickens [7]. It is therefore possible to speculate that the altered expression of tlps in 11168-GS may contribute to reduced ability of this variant to colonise animals and to invade mammalian cells in cell culture [11].

\section{Conclusion}

In conclusion, this study has demonstrated that chemoreceptor subsets vary between $C$. jejuni strains with the aspartate receptor, tlp1, conserved in all subsets observed. Expression of chemosensory group A tlp genes was similar between strains with $t l p 7$ and $t l p 10$ typically the highest expressed tlps and with expression generally higher in animal hosts than under laboratory conditions.

\section{Methods}

\section{C. jejuni strains and growth conditions}

C. jejuni strains NCTC 11168-GS, 11168-O (original Skirrow's isolate) and 81116 were kindly donated by D.G Newell (Veterinary Laboratory Agency, London, UK). Human isolates 173, 351, 430, 435, 440, 520, 705, 8, 193 and chicken isolates 019, 108,331, 434, 506, 008 and 193 were from RMIT/Griffith Universities culture collections, C. jejuni 81-176 was kindly donated by J. Fox, MIT, Boston, USA and C. jejuni GCH1-17 were collected between 19/01/2010 and 12/03/10 by S.K. Day from Queensland Health Pathology, Gold Coast Hospital, Queensland, Australia. Campylobacter cells were grown on solid selective agar (Columbia agar, 5\% (v/v) defibrinated horse blood, Skirrow Selective Supplement; Oxiod) under microaerobic conditions $\left(5 \% \mathrm{O}_{2}, 15 \%\right.$ $\mathrm{CO}_{2}, 80 \% \mathrm{~N}_{2}$; BOC gases) for 48 hours at $42^{\circ} \mathrm{C}$. C. jejuni was harvested from the agar plates in sterile Brucella Broth (BBL) and the cfu/mL was determined by measuring $\mathrm{OD}_{600 \mathrm{~nm}}$ and comparing to a standard growth curve. Cultures for RNA analysis were grown under the following conditions: Cultures that mimic environmental conditions were performed as previously described [12]. Cultures grown for laboratory conditions were grown at either 37 or $42^{\circ} \mathrm{C}$ as described in Day et al. (2009) and processed to minimise effects on RNA expression as per King et al. (2012) [12,21].

\section{PCR amplification of $C$. jejuni Group A t/p genes}

A single $10 \mu \mathrm{L}$ loop full of bacteria was removed from an agar plate covered with a confluent growth of the C. jejuni strains and resuspended in $1 \mathrm{~mL}$ of sterile water. The bacterial suspension was boiled for $5 \mathrm{~min}$ and the cell debris was pelleted by centrifugation at $13,000 \mathrm{~g}$, and the supernatant was used as template DNA for PCR analysis. The template DNA was used at $10 \%$ of the final PCR volume in the presence of 10 pmoles of forward and reverse primer (Table 2), $10 \mu \mathrm{M}$ dNTPs, $1 \mathrm{x}$ polymerase reaction buffer, 1 unit of thermal stable DNA polymerase and $3.5 \mathrm{mM} \mathrm{MgCl}$. The 
Table 2 Primers used in this study

\begin{tabular}{|c|c|}
\hline Primer nam & $5^{`}-3^{`}$ primer sequence \\
\hline Tlp1p F & TTG TTA TCG TIT ACG CTG ATG \\
\hline Tlp1p R & TGG AAG ATC TाT ATT ATA ATT TाT TAA GGG TाT AA \\
\hline Tlp2p F & CAT ATG CAA GCA ATT TाT CAT GAA GTT GTG A \\
\hline Tlp2p R & CTC GAG TTA TIT ATA AAC TGG AGC TTC TAT TTG Tा \\
\hline Tlp3p F & CAT ATG ACC TCA CTA TAT GAA AGC ACT CTT \\
\hline Tlp3p R & CTC GAG TTA TGC AGC TTT ATA AAT AGG TाT ATT TAT A \\
\hline Tlp4p F & CTC GAG GAT TCG AGA AAC AAT ACA TAT GAA TT \\
\hline Tlp4p R & CTC GAG TTA TTG TTT CAT TAA AAT AGA ATT AAC AGC \\
\hline Tlp7p F & CAT AGT TIT AAA AAT ACT GCC AAT AAA ATG AG \\
\hline Tlp7p R & CTC GAG TTA AGA TTG ACT GGT TIT GCT TAT ATC \\
\hline Tlp7i F & CTG CGA TCT CAT CCA TCA TTT GAG TTG C \\
\hline Tlp7i R & CAT GCT AAA GAA TTA GCT CAA GGA AGT GGC \\
\hline Tlp10p F & CAT ATG AAC TAT TCT TCA TCT AAA GAT AAT AA \\
\hline Tlp10p R & CTC GAG TTA TIT AAA TAA ATT AGA TTG TTC TAT AGT \\
\hline Tlp11mid F & CTC TGA TGG CAA AAG TGT AAC \\
\hline Tlp11mid R & CTC TTC AGA TTG AGC GAT AAC \\
\hline $\begin{array}{l}\text { Therm } 1 \\
\text { (23SRNA) }\end{array}$ & TTA TCC AAT ACC AAC ATT AGT \\
\hline $\begin{array}{l}\text { Therm } 2.1 \\
\text { (23SRNA) }\end{array}$ & GAA GAT ACG GTG CTA TTT TG \\
\hline
\end{tabular}

PCR reaction was performed as follows; $95^{\circ} \mathrm{C}$ for 5 mins for 1 repeat, $95^{\circ} \mathrm{C}$ for 30 seconds, $50^{\circ} \mathrm{C}$ for $1 \mathrm{mi}-$ nute and $72^{\circ} \mathrm{C}$ for 1 minute for 45 repeat cycles followed by a final extension of $72^{\circ} \mathrm{C}$ for 5 minutes. Presence of PCR product amplification was determined by agarose gel electrophoresis.

\section{Preparation of $C$. jejuni inoculum}

C. jejuni cells were harvested from Columbia agar plates in $1 \mathrm{~mL}$ of PBS $(137 \mathrm{mM} \mathrm{NaCl}, 2.7 \mathrm{mM} \mathrm{KCl}, 10 \mathrm{mM}$ $\mathrm{Na}_{2} \mathrm{HPO}_{4}$ and $1.8 \mathrm{mM} \mathrm{KH}_{2} \mathrm{PO}_{4}, \mathrm{pH}$ 7.5) and the concentration was adjusted to $1 \times 10^{8} \mathrm{cfu} / \mathrm{mL}$ using spectrophotometry followed by a viable count.

\section{Inoculation of chickens with C. jejuni}

Ross breed chickens (Barters, Rochdale, Qld), with maximum age difference of 2 hours and at one day after hatching, were placed into groups of five, colour marked and pre-inoculation faecal samples were taken from the cloaca and cultured. Chickens were housed in clean barrier cages at $28^{\circ} \mathrm{C}$ and allowed access to sterilised food and water. All experiments were approved by the Griffith University Animal Ethics Committee (approval number: MSC/04/08/AEC).

Following a pre-inoculation cloacal swab, one day old chickens were orally inoculated with $30 \mu \mathrm{L}$ PBS containing $1 \times 10^{8} \mathrm{cfu}$ bacterial cells as previously described [22]. On day 6 , euthanasia was performed by cervical dislocation. Post-mortem caecal samples were obtained by the dissection of the caeca aseptically. Whole $C$. jejuni cells were collected directly from the caeca with the use of antibody coated M-280 Dyna-beads as previously described [21].

\section{Inoculation of mice with C. jejuni}

Murine studies were performed using the 129X1/SvJ background male mice (Animal Resource Centre, Western Australia) aged between 6-8 weeks as previously described [23]. The mice were housed under clean conventional conditions in groups of 4-6, with free access to sterilised food and water. All experiments were approved by the Griffith University Animal Ethics Committee (Approval number: BDD/01/07).

Following a pre-inoculation swab, 129X1/SvJ mice were orally inoculated with $30 \mu \mathrm{L}$ PBS containing $1 \mathrm{x}$ $10^{8} \mathrm{cfu}$ of bacterial cells. After 48 hour post-inoculation, the animals were euthanised by cervical dislocation, and the gastrointestinal tissues, small and large intestine, were collected aseptically [23]. The contents of the intestines were removed and whole $C$. jejuni cells were isolated directly from the sample with the use of antibody coated M-280 Dyna-beads as previously described [21].

\section{Immunomagnetic separation (IMS) of $C$. jejuni from chicken and mouse intestinal content}

Immunomagnetic separation (IMS) of C. jejuni from chicken and mouse intestinal content was performed as previously described [21]. Briefly, intestinal content or caecal content was removed and Brucella Broth was added to a final volume of $2 \mathrm{~mL}$. After removal of debris, $80 \mu \mathrm{L}$ of anti-C. jejuni (Fitzgerald) coated M-280 Dyna-beads were added to the intestinal or caecal content and incubated with tilt rotation at $4^{\circ} \mathrm{C}$ for 30 mins. Dyna-beads were removed from the sample using IMS and washed three times with Isotonic PBS containing $0.1 \%$ tween-20 at $4^{\circ} \mathrm{C}$. Bound Campylobacter was eluted from the beads using $0.05 \%$ trypsin-EDTA (Invitrogen), supernatant was removed and centrifuged at 10,000 $\mathrm{x} g$ to yield a bacterial pellet. RNA was extracted using Qiagen RNase easy kit, with on-column DNase digestion.

\section{Primer design}

Primers were designed based on the published nucleotide sequence of $C$. jejuni 11168 [24] to allow PCR amplification of the periplasmic sensory domain of the group A $t l p$ receptors, $t l p 1-4,7$ and 10 . Tlp11 primers were designed on the sequence of tlp 11 from C. jejuni 520 (sequence not published) and the sequenced strain 8425 . Therm 1 and 2.1 primers, which amplify the $23 \mathrm{~s}$ RNA gene [25] were used as internal control. Primers used in this study are listed in Table 2. 


\section{Q RT-PCR analysis of tlp expression in C. jejuni}

Total RNA was extracted using RNeasy kit according to manufacturer's protocol (Qiagen) with on-column DNase. Extracted RNA was used as template for the reverse transcription reaction; $10 \mu \mathrm{L}$ of cDNA was synthesised by using gene specific primers (Table 2) and Improm II reverse transcriptase (Promega). All samples were reverse transcribed under the same conditions, $42^{\circ} \mathrm{C}$ for 1 hour, and the same reverse transcriptase mastermix, to reduce differences in RT efficiency.

Q RT-PCR was performed in $20 \mu \mathrm{L}$ with $1.5 \mu \mathrm{L}$ of cDNA, $10 \mu \mathrm{L}$ Sensimix (Quantace) and $250 \mathrm{nM}$ sense and anti-sense primers (Table 2). The qPCR reactions were carried out using a Bio-Rad iQ5 PCR machine and Bio-Rad iQ5 optical system software program.

All qPCR reactions were carried out using the same thermal profile conditions, $94^{\circ} \mathrm{C}$ for 5 minutes, then 45 cycles of $94^{\circ} \mathrm{C}$ for 30 seconds, $48^{\circ} \mathrm{C}$ for 30 seconds then $72^{\circ} \mathrm{C}$ for 1 minute, 30 seconds with fluorescence measured during the $72^{\circ} \mathrm{C}$ extension phase. Melt curves were produced for each amplification product and these were measured 80 times over the incremental increases in temperature. Amplification plots and melt curves were analysed by the Bio-Rad iQ5 optical system software program. Products were reconfirmed by performing agarose gel electrophoresis.

A PCR standard curve was generated for each primer set by performing five ten-fold serial dilutions. Quantity values (copies) for gene expression was generated by comparison of the fluorescence generated by each sample with a standard curve of known quantities for each PCR product. The standard curve equations are listed in Table 3.

\section{Immunisation of mice and production of polyclonal anti-sera}

Preimmune serum was collected prior to immunisation and tested for reactivity with $C$. jejuni and with purified Tlp1 protein. Five female BALB/c mice (SPF) were injected subcutaneously with a total volume of $200 \mu \mathrm{L}$ consisting of $50 \mu \mathrm{g}$ of His-tagged Tlp $1^{\text {peri }}$, expressed and

Table 3 PCR standard curves

\begin{tabular}{lcc}
\hline Gene & standard curve equation & efficiency \\
\hline TIp1 & $y=-3.764+42.062$ & $84.3 \%$ \\
TIp2 & $y=-3.670+37.969$ & $95 \%$ \\
Tlp3 & $y=-3.638+43.558$ & $88 \%$ \\
TIp4 & $y=-2.288+34.017$ & $173 \%$ \\
Tlp7 & $y=-3.486+45.126$ & $93.6 \%$ \\
TIp10 & $y=-3.641+45.241$ & $88.2 \%$ \\
TIp11 & $y=-5.297+60.289$ & $54.4 \%$ \\
23 S RNA & $y=-3.828+43.454$ & $82.1 \%$ \\
\hline
\end{tabular}

purified as previously described [7], combined with an equal volume of Freund's Incomplete adjuvant (Sigma) on day 0 . On days 14, 28 and 42 mice were boosted subcutaneously with $25 \mu \mathrm{g}$ of His-tagged-Tlp $1^{\text {peri }}$ combined with an equal volume of Freund's incomplete adjuvant (Sigma). A test-bleed was taken on day 35. On day 56, blood was harvested via cardiac puncture. Blood was allowed to clot at room temperature and the serum was collected for further use. The specificity of anti-Tlp1 ${ }^{\text {peri }}$ serum was verified by Western blot analysis and ELISA against cell lysates. All experiments were approved by the Griffith University Animal Ethics Committee (Approval number: BDD/01/09).

\section{Western blot analysis of Tlp1}

C. jejuni lysates of bacteria grown or maintained at room temperature, $37^{\circ} \mathrm{C}$ and $42^{\circ} \mathrm{C}$ were prepared by the harvesting of $10^{9}$ bacteria per $\mathrm{mL}$ in autoclaved water. $40 \mu \mathrm{L}$ of this suspension $\left(4 \times 10^{7}\right.$ C. jejuni) were mixed with SDS-PAGE loading buffer and boiled for 5 minutes and loaded onto the gel. SDS-PAGE and Western blot were performed as previously described [26] using a 1:200 dilution of the anti-Tlp1 $1^{\text {peri }}$ serum. Cell counts were verified to ensure equal number of bacteria was used in each well. Reactivity of the anti-sera to specific antigens was detected as previously described [7]. An anti-C. jejuni antibody (Fitzgerald) was also used to obtain a loading control. Briefly, the anti-C. jejuni antibody was used to screen blots of whole C. jejuni $\left(4 \times 10^{7}\right)$ maintained under the conditions listed above. All of the proteins bound by the antibody were analysed using QuantityOne software (Bio-Rad) to identify the one with the least variability between conditions and strains. A $\sim 30 \mathrm{kDa}$ protein was identified as the least variable with no significant change detected in expression between strains or growth conditions. This band was then used for the loading controls.

\section{Abbreviations}

Tlp: Transducer Like Protein; IMS: ImmunoMagnetic Separation.

\section{Competing interests}

The authors declare that they have no competing interests.

\section{Acknowledgements}

This work was funded by an NHMRC Project Grant. CJD was funded by a Griffith University Postdoctoral Fellowship.

\section{Author details}

${ }^{1}$ Institute for Glycomics, Griffith University Gold Coast Campus, Griffith University, Griffith, Australia. ²Department of Microbiology, Pathology Queensland, Gold Coast Hospital, Southport, Australia.

\section{Authors' contribution}

CJD Performed and planned experiments and wrote large portions of the final manuscript. LEHT Performed and planned experiments and wrote large portions of the final manuscript. LKS Produced antibody for analysis of Tlp1 and performed experiments utilising this antibody. Also helped in the preparation of the final manuscript. RMK Helped plan and performed animal 
work and helped prepare the final manuscript. GT Performed and planned many of the experiments involving Tlp11 and helped prepare the final manuscript. SKD Identified, isolated and provided fresh clinical isolates for this publication. EAS Helped perform animal work and preparation and performing of experiments involving GCH isolates and aided in the preparation of the final manuscript. VK Devising of initial experiment, planning of experiments and drafting of the manuscript. All authors read and approved the final manuscript.

\section{Received: 21 March 2012 Accepted: 29 June 2012}

Published: 29 June 2012

\section{References}

1. Friedman C, Neimann J, Wegener H, Tauxe R: Epidemiology of Campylobacter jejuni infections in the United States and other industrialized nations. In Campylobacter. 2nd edition. Edited by Nachamkin I, Blaser M. Washington DC: ASM Press; 2000:121-138.

2. Oosterom J, Butzler J: Campylobacter: pathogenicity and significance in foods. Int J Food Microbiol 1991, 12:1-8.

3. Young KT, Davis LM, DiRita VJ: Campylobacter jejuni: molecular biology and pathogenesis. Nat Rev Microbiol 2007, 5:665-679.

4. Josenhans $C$, Suerbaum $\mathrm{S}$ : The role of motility as a virulence factor in bacteria. Int J Med Microbiol 2002, 291(8):605-616.

5. Marchant J, Wren B, Ketley J: Exploiting genome sequence: predictions for mechanisms of Campylobacter chemotaxis. Trends Microbiol 2002, 10(4):155-159.

6. Korolik V, Ketley JM: Chemosensory signal transduction pathway of Campylobacter jejuni. In Campylobacter. thirdth edition. Edited by Nachamkin I, Symanski C, Blaser MJ. Washington, DC: ASM Press; 2008:351-366.

7. Hartley-Tassell LE, Shewell LK, Day CJ, Wilson JC, Sandhu R, Ketley JM, Korolik V: Identification and characterization of the aspartate chemosensory receptor of Campylobacter jejuni. Mol Microbiol 2009

8. Tareen AM, Dasti Jl, Zautner AE, Gross U, Lugert R: Campylobacter jejuni proteins Cj0952c and Cj0951c affect chemotactic behaviour towards formic acid and are important for invasion of host cells. Microbiology 2010, 156(Pt 10):3123-3135.

9. Lane M, Lloyd A, Markyvech T, Hagan E, Mobley H: Uropathogenic Escherichia coli strains generally lack functional Trg and Tap chemoreceptors found in the majority of E. coli strains residing in the gut. J Bacteriol 2006, 188:5618-5625.

10. Zautner AE, Herrmann S, Corso J, Tareen AM, Alter T, Gross U: Epidemiological association of different Campylobacter jejuni groups with metabolism-associated genetic markers. Appl Environ Microbiol 2011, 77(7):2359-2365.

11. Gaynor EC, Cawthraw S, Manning G, MacKichan JK, Falkow S, Newell DG: The Genome-Sequenced Variant of Campylobacter jejuni NCTC 11168 and the Original Clonal Clinical Isolate Differ Markedly in Colonization, Gene Expression, and Virulence-Associated Phenotypes. J Bacteriol 2004, 186(2):503-517.

12. Day CJ, Tiralongo J, Hartnell RD, Logue CA, Wilson JC, von Itzstein M, Korolik V: Differential carbohydrate recognition by Campylobacter jejuni strain 11168: influences of temperature and growth conditions. PLOS One 2009, 4(3):e4927.

13. Blaser MJ, Cody HJ: Methods for isolating Campylobacter jejuni from lowturbidity water. Appl Environ Microbiol 1986, 51(2):312-315.

14. Craun GF, Brunkard JM, Yoder JS, Roberts VA, Carpenter J, Wade T, Calderon RL, Roberts JM, Beach MJ, Roy SL: Causes of outbreaks associated with drinking water in the United States from 1971 to 2006. Clin Microbiol Rev 2010, 23(3):507-528,

15. Kemp R, Leatherbarrow AJ, Williams NJ, Hart CA, Clough HE, Turner J, Wright EJ, French NP: Prevalence and genetic diversity of Campylobacter spp. in environmental water samples from a 100-square-kilometer predominantly dairy farming area. Appl Environ Microbiol 2005, 71(4): 1876-1882.

16. Newell DG, McBride H, Saunders F, Dehele Y, Pearson AD: The virulence of clinical and environmental isolates of Campylobacter jejuni. J Hyg (Lond) 1985, 94(1):45-54.

17. Guccione E, Leon-Kempis Mdel R, Pearson BM, Hitchin E, Mulholland F, van Diemen PM, Stevens MP, Kelly DJ: Amino acid-dependent growth of Campylobacter jejuni: key roles for aspartase (AspA) under microaerobic and oxygen-limited conditions and identification of AspB (Cj0762), essential for growth on glutamate. Mol Microbiol 2008, 69(1):77-93.

18. Leon-Kempis Mdel R, Guccione E, Mulholland F, Williamson MP, Kelly DJ: The Campylobacter jejuni PEB1a adhesin is an aspartate/glutamatebinding protein of an $A B C$ transporter essential for microaerobic growth on dicarboxylic amino acids. Mol Microbiol 2006, 60(5):1262-1275.

19. Hazelbauer GL, Engstrom P, Harayama S: Methyl-accepting chemotaxis protein III and transducer gene trg. J Bacteriol 1981, 145(1):43-49.

20. Blaser M, Perez G, Smith P, Patton C, Tenover F, Lastovica A, Wang W: Extraintestinal Campylobacter jejuni and Campylobacter coli infections: host factors and strain characteristics. J Infect Dis 1986, 153(3):552-559.

21. King RM, Day CJ, Hartley LE, Connerton IF, Tiralongo J, McGuckin MA, Korolik V: Carbohydrate binding and gene expression by in vitro and in vivo propagated Campylobacter jejuni after Immunomagnetic Separation. J Basic Microbiol 2012, doi:10.1002/jobm.201100466. In Press.

22. Ringoir DD, Szylo D, Korolik V: Comparison of 2-day-old and 14-day-old chicken colonization models for Campylobacter jejuni. FEMS Immunol Med Microbiol 2007, 49(1):155-158.

23. McAuley JL, Linden SK, Png CW, King RM, Pennington $\mathrm{HL}$, Gendler SJ, Florin TH, Hill GR, Korolik V, McGuckin MA: MUC1 cell surface mucin is a critical element of the mucosal barrier to infection. J Clin Invest 2007, 117(8):2313-2324.

24. Parkhill J, Wren BW, Mungall K, Ketley JM, Churcher C, Basham D, Chillingworth T, Davies RM, Feltwell T, Holroyd S, et al: The genome sequence of the food-borne pathogen Campylobacter jejuni reveals hypervariable sequences. Nature 2000, 403(6770):665-668.

25. Eyers M, Chapelle S, Van Camp G, Goossens H, Wachter RD: Discrimination among thermophilic Campylobacter species by polymerase chain reaction amplification of 23 S rRNA gene fragments. J Clin Microbiol 1994, 32(6):1623.

26. Sambrook J, Fritsch EF, Maniatis T: Molecular cloning: a laboratory manual. 2nd edition. Cold Spring Harbor, N.Y: Cold Spring Harbor Laboratory Press; 1989.

doi:10.1186/1471-2180-12-128

Cite this article as: Day et al: Variation of chemosensory receptor content of Campylobacter jejuni strains and modulation of receptor gene expression under different in vivo and in vitro growth conditions. BMC Microbiology 2012 12:128.

\section{Submit your next manuscript to BioMed Central and take full advantage of:}

- Convenient online submission

- Thorough peer review

- No space constraints or color figure charges

- Immediate publication on acceptance

- Inclusion in PubMed, CAS, Scopus and Google Scholar

- Research which is freely available for redistribution 